\title{
Indentation haute fréquence : vers le contrôle non-destructif des structures
}

\author{
Bruno Passilly $^{1 a}$, Benjamin Lamboul ${ }^{1}$ et Jean-Michel Roche ${ }^{1}$
}

Reçu le 8 novembre 2016, accepté le 24 mars 2017

\begin{abstract}
Résumé - La nanoindentation est couramment utilisée pour déterminer les propriétés mécaniques locales des matériaux. La matière est sollicitée de façon quasi statique en appliquant un indenteur sur la surface à analyser. À partir de la courbe représentant la charge appliquée par l'indenteur sur le matériau en fonction du déplacement de l'indenteur, les modèles classiques permettent de déterminer le module d'Young local en tout point de test [Oliver \& Pharr, AIP Conference proceedings 7 (1992) 1564-1583; Doerner \& Nix, J. Mater. Res. 1 (1986) 601-609; Loubet et al., Vickers indentation curves of elastoplastic materials, in American Society for Testing and Materials STP 889, Microindentation Techniques in Materials Science and Engineering, Blau \& Lawn eds, 1986, pp. 72-89]. Cet essai est surtout utilisé sur de petites surfaces de matière $\left(<1 \mathrm{~cm}^{2}\right)$, qui doivent présenter un état de surface poli et plan afin de ne pas fausser la mesure, mais n'est pas adapté sur des pièces de structure de type tôle ou sandwich composite $\left(>1000 \mathrm{~cm}^{2}\right)$. Par extension de la méthode CSM (Continuous Stiffness Measurement) [Asif et al., Rev. Sci. Instrum. 70 (1999) 2408-2413], l'indenteur peut servir de générateur de vibrations. Pour cela l'indenteur est positionné sur un empilement de céramiques piézoélectriques et est appliqué sur la surface à analyser à une charge fixe de $1000 \mathrm{mN}$. L'indenteur est soumis à une oscillation à une fréquence de $5 \mathrm{kHz}$, alimenté à $10 \mathrm{~V}$. Les ondes ultrasonores ainsi générées, dites « ondes de Lamb», induisent un déplacement nanométrique de la surface, détectable par un vibromètre laser. Il est alors possible de suivre la propagation du front d'onde et de détecter ses interactions avec d'éventuels défauts de la structure inspectée [Boro Djordjevic, Quantitative ultrasonic guided wave testing of composites, The 39th Annual Review of Progress, 2013]. Il en résulte une cartographie complète de la surface. L'indenteur peut aussi être utilisé comme récepteur de l'onde générée. Le positionnement d'indenteurs récepteurs en plusieurs endroits de la structure permet de mesurer le temps de vol de l'onde entre l'indenteur émetteur et l'indenteur récepteur. La connaissance précise de la distance entre les points d'émission et de réception de l'onde permet de mesurer les vitesses en fonction de l'anisotropie du matériau, ce qui, à terme, peut permettre de remonter à ses constantes d'élasticité.
\end{abstract}

Mots clés : Pyramide Vickers / Indentation haute fréquence / Contrôle Non-Destructif / ondes guidées / vibrométrie Laser / mesure de temps de vol

\begin{abstract}
Frequency indentation: towards Non Destructive Test of structures. Nanoindentation is commonly used to determine local mechanical properties of materials. The material is tested with a load which is quasi-statically applied by an indenter, on the surface to be characterized. From the load/displacement curve, classic analytical models enable to determine Young modulus on every performing test point [Oliver \& Pharr, AIP Conference proceedings 7 (1992) 1564-1583; Doerner \& Nix, J. Mater. Res. 1 (1986) 601-609; Loubet et al., Vickers indentation curves of elastoplastic materials, in American Society for Testing and Materials STP 889, Microindentation Techniques in Materials Science and Engineering, Blau \& Lawn eds, 1986, pp. 72-89]. This test is appropriate only for small surface of materials $\left(<1 \mathrm{~cm}^{2}\right)$ which has to be well polished and planned to have the best measurements but is not suitable for structural parts like sheet metal or composite sandwich $\left(>1000 \mathrm{~cm}^{2}\right)$.
\end{abstract}

\footnotetext{
a Auteur de correspondance : bruno.passilly@onera.fr

1 Département Matériaux et Structures Composites, ONERA, 29 avenue de la division Leclerc, 92322 Chatillon Cedex, France
} 
Through extension of the CSM (Continuous Stiffness Measurement) method [Asif et al., Rev. Sci. Instrum. 70 (1999) 2408-2413], the indenter can also be used as vibrations generator. It is positioned on a piezoelectric stack and is applied on the surface to analyze with a contact load of $1000 \mathrm{mN}$. It is then submitted to an oscillating frequency of $5 \mathrm{kHz}$ under 10 Volts. Generated Lamb waves are received by a Laser vibrometer which is able to scan the material surface and to localize the impact damage of the structure [Boro Djordjevic, Quantitative ultrasonic guided wave testing of composites, The 39th Annual Review of Progress, 2013]. The indenter can also be used as the receiver of the generated wave. Receiver indenters are positioned in several locations in the structure to allow the measurement of time of flights between the emitting indenter and the receiver ones. The measurement of the distances between the emission point and the different reception points is required to measure the wave velocities and ultimately characterize the anisotropy of the metal sheet. Further studies could even lead to the assessment of the elastic properties.

Key words: Vickers indenter / high frequency indentation / Non Destructive Test / Guided waves / Laser Vibrometry / Time of flight

\section{Introduction}

La nanoindentation est une technique couramment utilisée pour déterminer les propriétés mécaniques locales des matériaux. L'indenteur est appliqué sur la surface du matériau de façon quasi-statique et on mesure la charge appliquée par l'indenteur sur le matériau en fonction du déplacement de l'indenteur dans le matériau. Cette méthode permet d'extraire le module d'Young local du matériau à caractériser d'après des modèles analytiques classiques [1-3]. Or, pour l'inspection et la caractérisation non-destructives de plaques de matériaux composites ou de tôles de grande dimension $\left(300 \times 300 \mathrm{~mm}^{2}\right)$, cette méthode n'est pas suffisante puisqu'il faudrait réaliser des milliers d'essais pour cartographier toute la pièce.

Dans le domaine du CND (contrôle non-destructif), il est courant d'avoir recours à des techniques d'imagerie basées sur la propagation d'ondes ultrasonores guidées, dites « ondes de Lamb», excitées typiquement par des disques piézoélectriques collés sur la structure ou fixés à l'aide de gels couplants. La reconstruction du champ vibratoire se fait par exemple au moyen d'un vibromètre Laser à effet Doppler. La signature des éventuels défauts présents dans la structure correspond à leur interaction avec l'onde, qui se traduit par une accumulation locale d'énergie vibratoire, comme cela a déjà été illustré pour des tôles ou sandwichs composites [5-7]. Notons qu'il est également possible d'utiliser une caméra infrarouge, plutôt qu'un vibromètre, pour détecter les défauts, dont la présence est cette fois détectée par l'échauffement local qu'ils créent (technique de vibrothermographie) [8-10]. De plus, il est également possible de mesurer les vitesses de propagation des ondes dans plusieurs directions, afin de caractériser l'anisotropie de l'échantillon, voire de remonter à ses constantes d'élasticité.

De telles méthodes ne permettent cependant pas d'inspecter ou de caractériser des surfaces courbes car le collage n'est pas possible et le gel couplant est inapproprié. En outre, il est difficile de contrôler la force d'appui de la pastille piézoélectrique sur la pièce et de la déplacer d'un endroit à l'autre de la pièce. D'où l'idée de remplacer ces excitateurs par des indenteurs pyramidaux, couramment utilisés en nanoindentation, qui, eux, peuvent être appliqués indifféremment sur une surface plane ou courbe, et dont l'emploi peut être détourné pour générer des vibrations. Adaptables sur des surfaces de grande dimension, leur force d'appui sur la surface du matériau à caractériser est, de plus, bien contrôlée, ce qui confère à l'essai un caractère plus maîtrisé et reproductible. Le balayage en fréquence, nécessaire pour localiser l'ensemble des défauts d'une structure lorsque ceux-ci sont de dimensions variables, est également possible en envoyant un signal de type burst de fréquence variable sur l'indenteur.

Le présent article a pour but d'introduire un système innovant d'excitation d'ondes, dérivé de la technique de nanoindentation (Sects. 1 et 2), qui, couplé à un vibromètre Laser, permet la détection de défauts dans un échantillon (Sect. 3) et qui, de façon autonome, fournit des informations de nature à caractériser le matériau d'un point de vue mécanique (Sect. 4).

\section{De l'indentation classique a l'indentation haute frequence}

L'essai de dureté consiste à appliquer un pénétrateur de géométrie connue comme par exemple les indenteurs Vickers à géométrie pyramidale à base carrée, les indenteurs Berkovitch à géométrie pyramidale à base triangulaire, ou les indenteurs Brinell qui sont des indenteurs sphériques. Les indenteurs sont considérés comme indéformable et sont généralement en diamant, qui est le matériau le plus dur qui existe. Lorsque l'indenteur est appliqué sur un matériau à une charge fixée, une empreinte est observée après retrait de la charge. En mesurant les dimensions de l'empreinte rémanente, et en connaissant la géométrie de l'indenteur et la charge maximale appliquée, il est possible de déterminer la dureté $H$ du matériau sollicité en calculant le rapport de la charge maximale $P$ appliquée sur l'aire de contact projetée $A_{c}$ qui dépend de la forme géométrique de l'indenteur.

$$
H=\frac{P}{A_{c}}
$$

Ces essais sont très utilisés pour une caractérisation macroscopique des matériaux, mais pour la caractérisation 


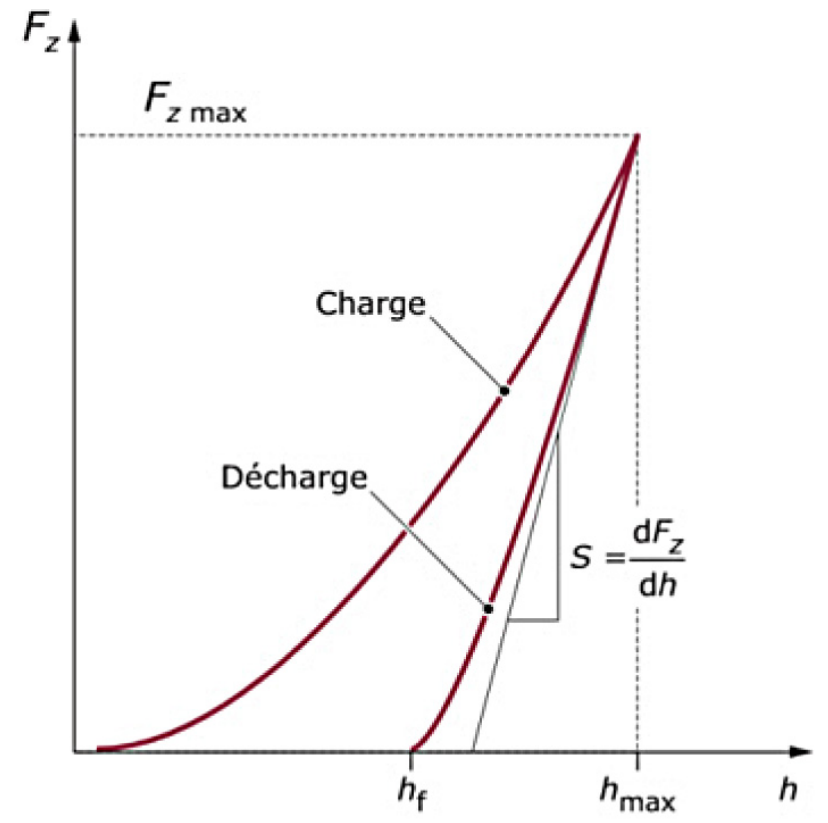

Fig. 1. Courbe classique d'indentation quasi-statique.

Fig. 1. Standard quasi-static indentation curve.

de revêtements par exemple, il est nécessaire d'appliquer des charges très faibles pour ne pas déformer le substrat, rendant de ce fait la mesure de l'empreinte extrêmement difficile avec les moyens de microscopie optique classique. Les moyens de nanoindentation ont alors fait leur apparition et consistent à appliquer de la même façon que précédemment un indenteur de géométrie connue sur le matériau à caractériser, mais la force appliquée par l'indenteur sera mesurée en continu ainsi que son déplacement associé pendant toute la durée de l'essai. Initialement, des essais quasi-statiques ont été réalisés afin d'obtenir une courbe caractéristique d'indentation (Fig. 1). Cette courbe est classiquement composée de deux parties distinctes. La première partie est la courbe de charge considérée généralement comme étant représentative d'un contact élasto-plastique, et correspond à la pénétration de l'indenteur dans le matériau jusqu'à la charge $F_{z \max }$ en décrivant une loi en puissance. La courbe de décharge constitue la deuxième partie de la courbe, le contact entre l'indenteur et le matériau est considéré comme purement élastique au début de la décharge. Cette courbe de décharge correspond au relèvement de l'indenteur en laissant à charge nulle une empreinte résiduelle de profondeur $h_{f}$.

La pente à la décharge permet de déterminer le module d'élasticité réduit de contact $E_{c}^{\prime}$ de la zone testée, calculée à partir de la connaissance de la raideur de contact $S$ et de l'aire projetée $A c$, selon l'expression suivante $[1,3]$ :

$$
E_{c}^{\prime}=\frac{S}{2} \sqrt{\frac{\pi}{A c}}
$$

La raideur de contact $S$ peut être déterminée à partir de la courbe de décharge en déterminant la tangente à la courbe au tout début de la décharge. Dans le cas d'une décharge non-linéaire, des approches ont été menées par différents auteurs pour considérer la décharge comme une loi en puissance dont la dérivée à charge maximale permettra de déterminer la raideur $S[2,3]$. La formation de bourrelets ou au contraire d'affaissements autour de l'empreinte rémanente est prise en compte dans ces modèles pour corriger la hauteur réelle de contact.

Le module de Young $E_{\text {mat }}$ du matériau testé est alors calculé en introduisant le module de l'indenteur $E_{\text {ind }}$ et les coefficients de Poisson de l'indenteur $\nu_{\text {ind }}$ et du matériau testé $\nu_{\text {mat }}$ suivant la relation :

$$
\frac{1}{E_{c}^{\prime}}=\frac{1-\nu_{m a t}^{2}}{E_{m a t}}+\frac{1-\nu_{i n d}^{2}}{E_{i n d}}
$$

Sur la base de ce principe, la méthode CSM (Continuous Stiffness Measurement) a été développée pour pouvoir mesurer la raideur de contact en continu pendant l'essai. Cette méthode consiste à surimposer pendant l'application de la charge de l'indenteur sur l'échantillon, une oscillation de la charge à une fréquence de quelques Hertz [4]. Sur la base de cette mise en oscillation de l'indenteur, Guillonneau établit une nouvelle méthode d'exploitation pour mesurer des modules d'élasticité de différents matériaux, comme le PMMA ou la silice, en ajoutant une fréquence d'oscillation de l'indenteur de 30 à $100 \mathrm{~Hz}$ [11]. Une fréquence plus élevée à très faible charge peut engendrer une perte du contact entre l'indenteur et le matériau et la conception actuelle des moyens de nanoindentation ne permet pas de générer les fréquences d'oscillation supérieures à $300 \mathrm{~Hz}$.

En réalisant un montage spécifique qui permette d'imposer une charge de $1000 \mathrm{mN}$ sur l'échantillon, et en réalisant une oscillation de l'indenteur de l'ordre de $5000 \mathrm{~Hz}$, l'indenteur restera en contact permanent avec l'échantillon. En dépassant la fréquence d'oscillation du mode CSM, la vibration de l'indenteur permettra de générer des ondes à la surface du matériau en réalisant une seule empreinte. L'information à traiter ne viendra pas de la mesure du déplacement associé à la variation de la force, mais de la mesure de la déformation de la surface de l'échantillon engendré par la propagation de l'onde sur toute la surface de l'échantillon.

\section{Démarche expérimentale}

Dans notre étude, l'indenteur en diamant est de type Vickers avec un angle entre faces de $136^{\circ}$. Il est fixé sur un empilement de disques piézoélectriques Physik Instrumente dont la référence est PICA P010.20. Le stack peut fournir un déplacement de $30 \mu \mathrm{m}$ pour une tension maximale de $1000 \mathrm{~V}$. Cet ensemble est maintenu sur un capteur de force Omega LCM 201. Une empreinte est réalisée par l'application de la pyramide sur la surface de l'échantillon à l'aide d'un translateur manuel qui permet de régler la force d'appui (Fig. 2). La géométrie de l'indenteur permet de générer des ondes sans directions de propagation préférentielles. Le mouvement alternatif 


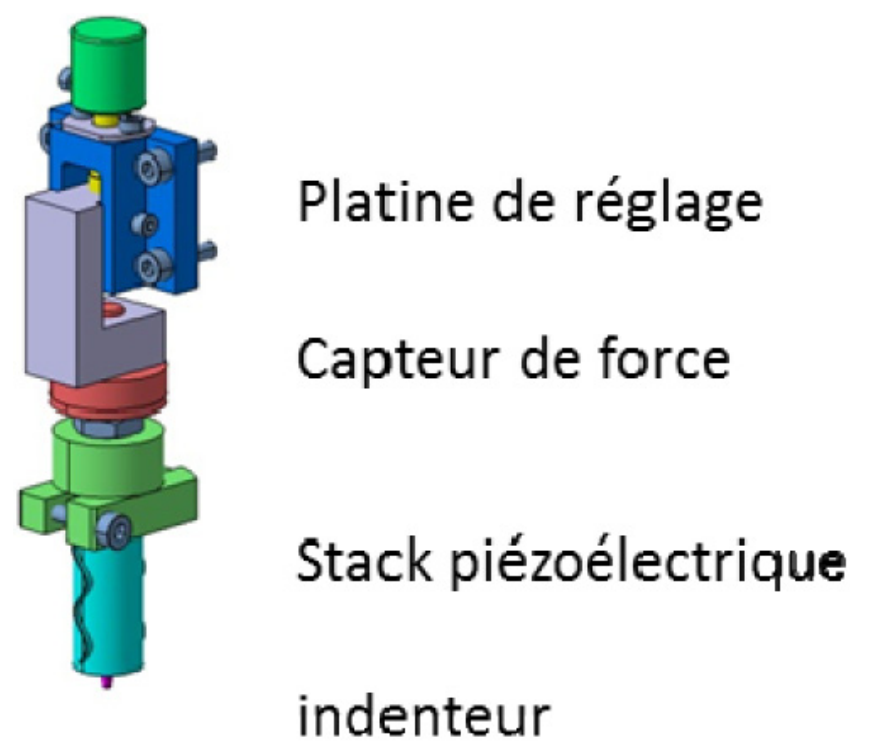

Fig. 2. Indenteur excitateur piézoélectrique muni d'un capteur et d'un translateur permettant de mesurer et contrôler sa force d'appui appliquée à la structure.

Fig. 2. Piezoelectric wave-generator indenter with a load sensor and a mechanical translator designed to monitor the load applied to the structure.

de la pointe génère des ondes de surface qui sont circulaires autour du point de contact. Le signal d'excitation du stack piézoélectrique est sinusoïdal, de type « burst », à une fréquence de $5 \mathrm{kHz}$ et une amplitude de $10 \mathrm{~V}$. A cette fréquence les ondes ultrasonores générées sont des ondes de flexion (mode $\mathrm{A}_{0}$ de Lamb). Chaque « burst » est répété toutes les 200 millisecondes.

Le scan de l'échantillon étudié est effectué au moyen du vibromètre Polytec OFV-505 LDV monopoint qui peut fonctionner jusqu'à une fréquence d'acquisition de $350 \mathrm{kHz}$. La tête optique OFV-505 utilise la longueur d'onde $633 \mathrm{~nm}$, et présente la particularité de disposer des fonctions autofocus et enregistrement du focus. La tête dispose de différentes lentilles pour s'adapter à la distance de l'objet à caractériser et permet d'adapter la taille du spot (de quelques dizaines de micromètres). Couplée avec le contrôleur OFV-5000, cette tête optique assure un traitement analogique/numérique haute résolution.

Le faisceau laser monopoint est dirigé sur un ensemble de deux miroirs oscillants (Fig. 3), ce qui rend possible le scan de la surface de la pièce à contrôler. Les miroirs optiques sont montés chacun sur l'axe d'un moteur galvanométrique. Chaque axe du scanner galvanométrique CTI a une possibilité de rotation de $+/-20^{\circ}$ (référence $8340 \mathrm{KM} 40 \mathrm{~A}$ ) et chacun est muni d'une commande avec étage de puissance (référence 67134HHJ-1HP). Les deux miroirs XY (référence 6M2430S40-S1) de 30 mm d'ouverture utile, sont traités à l'argent sur un substrat silicium. Un détecteur de courant permet de réguler la position angulaire du miroir et l'ensemble est intégré dans une boucle de régulation. Les miroirs sont pilotés en rotation pour balayer la surface suivant $\theta_{x}$ et $\theta_{y}$.
Pour chaque position de scan, le signal temporel est enregistré en quelques millisecondes par l'intermédiaire d'un oscilloscope Lecroy Wave Runner 44MXiA qui sert de carte d'acquisition et qui stocke les données (temps, tension) en un point donné de la zone à analyser. Cette acquisition sera renouvelée sur chaque point de la zone à analyser en envoyant toujours le même signal d'excitation sur la structure et en mesurant le signal réceptionné en chaque point de scan. Le résultat consistera à produire un film représentant temporellement l'image de l'ensemble de la surface de la zone scannée en fonction du temps. La Figure 6 est constituée de plusieurs images issues du film réalisé. En fonction des dimensions de la zone à inspecter et du pas de déplacement pour accroître la résolution, la durée d'acquisition des scans peut s'étendre de quelques minutes jusqu'à plusieurs heures.

Par extension de la méthode, comme l'illustre le paragraphe 4 , la détermination des temps de vol des ondes qui se propagent dans la structure ne nécessite pas de vibromètre Laser. Il est possible d'utiliser une pointe Vickers instrumentée comme explicité ci-dessus pour jouer le rôle de récepteur de l'onde émise par l'émetteur. Le montage expérimental proposé ici, représenté sur la Figure 4, est constitué d'un indenteur émetteur et de trois indenteurs récepteurs, positionnés au sommet d'un carré de $300 \times 300 \mathrm{~mm}^{2}$ (Fig. 7). Chaque indenteur Vickers est équipé comme précédemment d'un capteur de force, d'une platine de translation, et d'un stack piézoélectrique. Ces quatre ensembles sont appliqués sur la plaque à caractériser et le stack piézoélectrique émetteur est excité de la même façon que décrit plus haut. L'onde émise est recueillie tour à tour par chacun des trois autres indenteurs récepteurs. Il est ensuite possible de réitérer les mêmes mesures en excitant tour à tour chaque stack piézoélectrique afin de déterminer l'ensemble des temps de vols dans toutes les directions offertes par les quatre stacks. L'exploitation de ces données permet de façon immédiate de quantifier l'anisotropie des échantillons et peut mener jusqu'à l'estimation de certaines de leurs constantes d'élasticité.

\section{Application « détection de défauts *}

La démonstration de la pertinence de l'excitation d'ondes guidées par des nanoindenteurs instrumentés est effectuée pour un panneau sandwich fibres de verre / mousse Rohacell, impacté mécaniquement à $10 \mathrm{~J}$. L'âme en mousse, de $4 \mathrm{~mm}$ d'épaisseur, est recouverte de chaque côté par une peau en fibre de verre/époxy. La peau fine a une épaisseur de $2 \mathrm{~mm}$ et la peau épaisse a une épaisseur de $5 \mathrm{~mm}$. L'endommagement attendu correspond à un décollement entre la peau et la mousse, soit une perte de rigidité locale. Ce cas d'étude est choisi spécifiquement car les techniques de CND conventionnelles, telles que le scan ultrasonore et la thermographie infrarouge active, ne permettent pas la détection de ce type de décollement face opposée, en raison de la présence de la mousse [12].

Le panneau sandwich est sollicité par un indenteur positionné comme indiqué sur la Figure 5. L'effort appliqué 


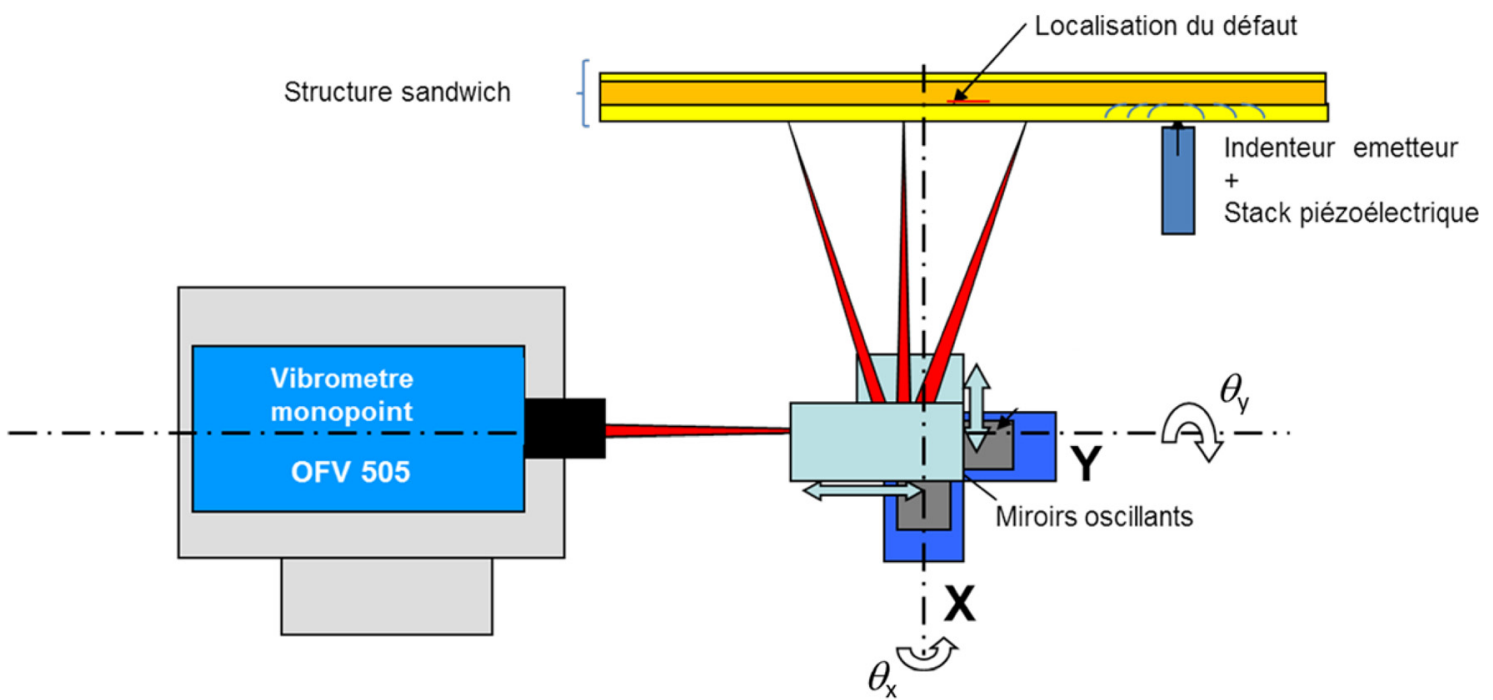

Fig. 3. Banc de vibrométrie Laser constitué du vibromètre monopoint et d'un système de miroirs oscillants permettant le scan complet de la structure à inspecter ou à caractériser.

Fig. 3. Laser vibrometer bench constituted of a monopoint vibrometer and a system of oscillating mirrors designed to scan or characterize the whole structure.

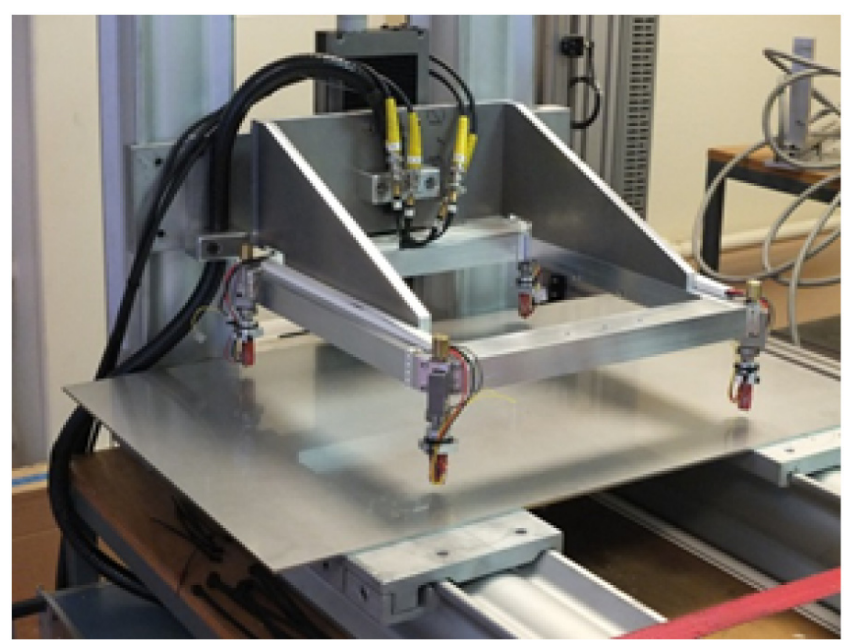

Fig. 4. Banc de quatre stacks piézoélectriques pour la caractérisation ultrasonore des structures.

Fig. 4. Four piezoelectric stack bench for ultrasonic characterization.

est associé à une empreinte de $1000 \mathrm{mN}$. La zone scannée est un carré de $130 \times 130 \mathrm{~mm}^{2}$, avec un pas de l'ordre de $2 \mathrm{~mm}$, ce qui correspond à 4200 acquisitions pour former une image temporelle de la surface à analyser. En tenant compte des temps d'acquisition, du déplacement des miroirs et de sauvegarde des données, le temps de scan est estimé à $2000 \mathrm{~s}$, soit environ une demi-heure. L'ensemble des données recueillies par le vibromètre permet d'explorer temporellement la propagation de l'onde à la surface de l'échantillon (Fig. 6). L'onde émise à partir de l'indenteur commence à interagir avec le défaut dès $62 \mu \mathrm{s}$. Puis cette interaction continue à être active même après le passage de l'onde à 88 et $122 \mu$ s. L'onde se

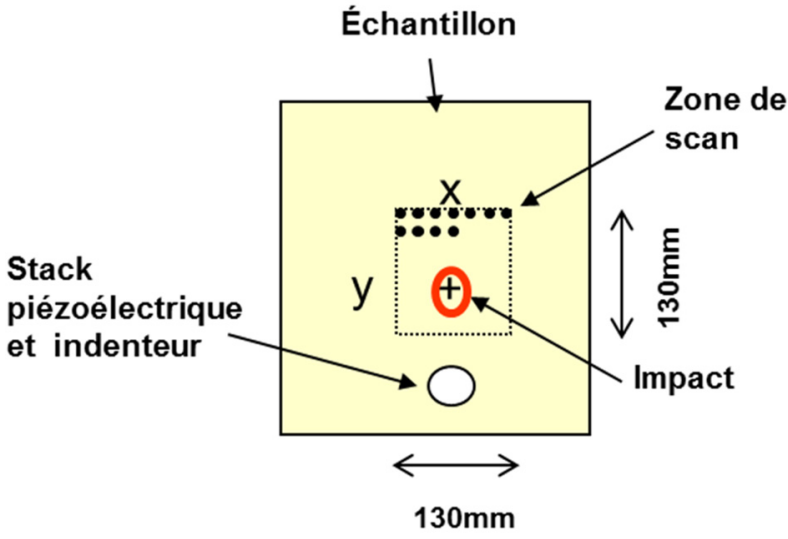

Fig. 5. Visualisation de la zone de scan, de l'emplacement de l'indenteur et du point d'impact.

Fig. 5. Schematic view of the scanned area, the indenter location and the impact point.

réfléchit ensuite sur les bords de la plaque sandwich et le défaut est alors « réactivé » par les ondes réfléchies, vers $195 \mu$ s. Au-delà de cet intervalle de temps, les réflexions multiples des ondes rendent le champ vibratoire difficilement interprétable; de plus, l'énergie propagée n'est plus assez importante pour continuer à entretenir l'excitation du défaut.

L'analyse des cartographies de vitesses instantanées prises à 122 et $195 \mu$ s permet l'identification d'un défaut dont l'étendue géométrique est de l'ordre de $20 \mathrm{~mm}$. La démonstration est ainsi faite qu'il n'est donc pas nécessaire de coller des disques piézoélectriques sur la structure et que l'effort appliqué par l'indenteur est suffisant pour propager l'onde dans le matériau. Une perspective immédiate serait d'étendre ce type d'essais à l'ins- 

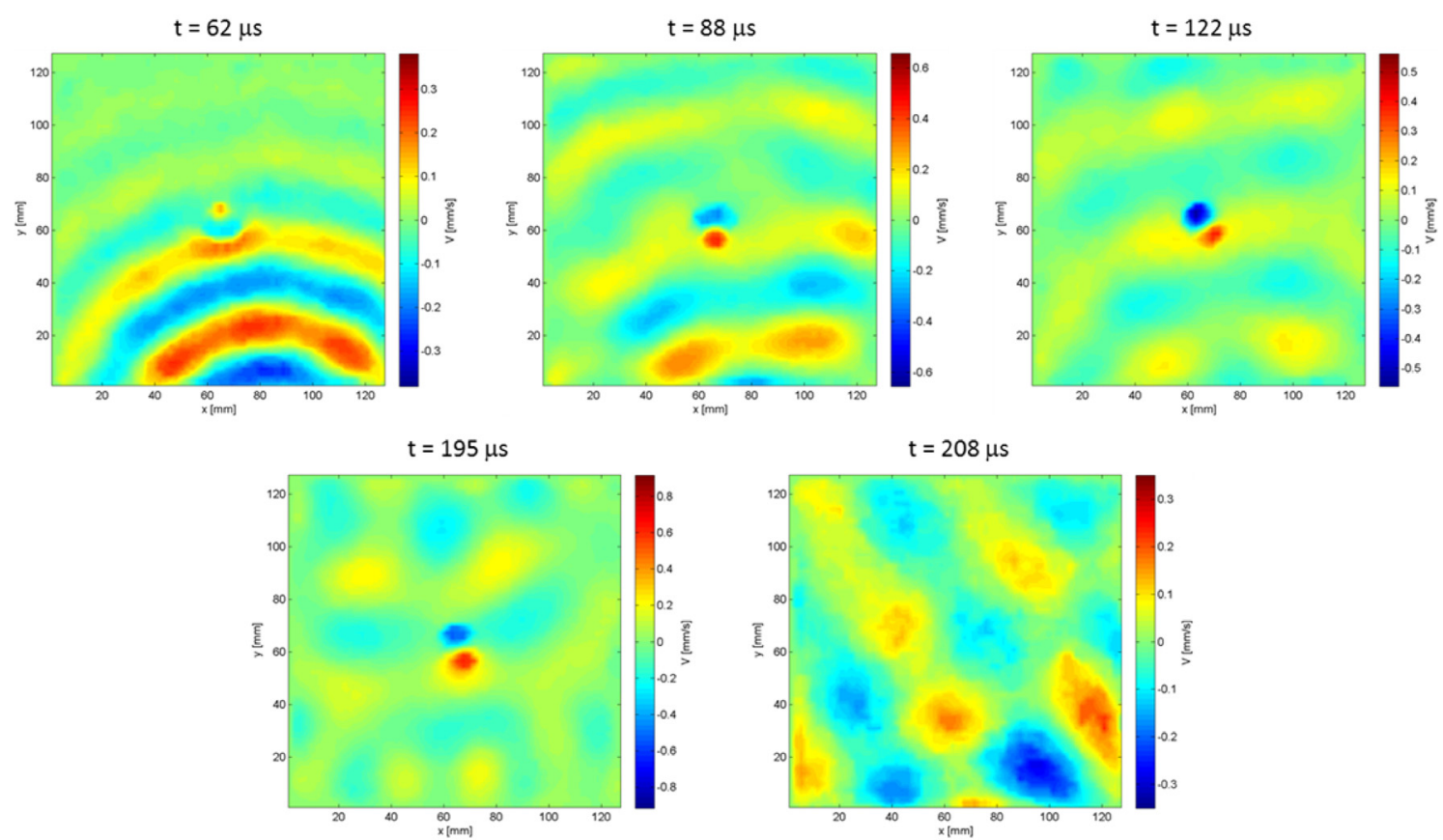

Fig. 6. Suivi temporel de la propagation des ondes guidées émises par un indenteur de type pyramidal sous une charge de $1000 \mathrm{mN}$ dans un panneau sandwich mousse impacté à $10 \mathrm{~J}$. Cartographies en vitesses. Scans $130 \times 130 \mathrm{~mm}^{2}$.

Fig. 6. Real-time monitoring of guided waves generated in a foam sandwich panel by a pyramidal indenter under a 1000 mN load. Detection of a $10 \mathrm{~J}$ impact. Velocity maps in a $130 \times 130 \mathrm{~mm}^{2}$. scanned area.

pection de pièces présentant des états de surface variés, y compris courbées, comme par exemple dans les structures aéronautiques.

\section{Application « caractérisation ultrasonore »}

Ce paragraphe a pour but de valider la pertinence du dispositif «quadripôle»présenté à la Figure 4 à des fins de caractérisation, et non plus simplement de détection de défauts. La démonstration est réalisée sur des structures de type tôles, en aluminium et en laiton. Le principe mis en avant est donc de produire des ondes de surface avec un des quatre indenteurs piézoélectriques et de collecter le signal sur les trois autres indenteurs, qui se comportent alors comme des récepteurs. La mesure des temps de vol et des vitesses de propagation sont comparées suivant les différentes directions de propagation. La convention de numérotation est la suivante : chacun des quatre stacks piézoélectriques est numéroté de 1 à 4 . L'indenteur émetteur est nommé $\mathrm{E}$, et les trois récepteurs sont nommés R. Ainsi, E2R3 correspond à une onde émise par le stack 2 et réceptionnée par le stack 3 . La tôle à caractériser sera donc sollicitée en $\mathrm{E} 1$ avec réception de l'onde émise en R2, R3, R4. Les combinaisons E2 R1 R3 R4, puis E3 R1 R2 R4, puis E4 R1 R2 R3 sont obtenues en excitant respectivement les indenteurs 2, 3 et 4 (Fig. 7).

La tôle est sollicitée avec l'envoi d'un « burst » d'une amplitude de $10 \mathrm{~V}$, à une fréquence de $5 \mathrm{kHz}$ sur l'un des quatre stacks piézoélectriques. Chaque «burst » est envoyé avec une répétabilité de 200 ms. L'échantillonnage

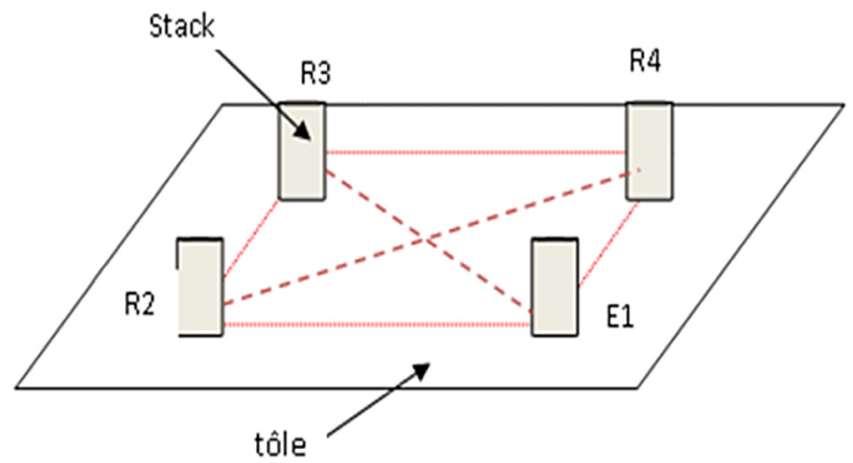

Fig. 7. Convention de numérotage du quadripôle à 4 stacks piézoélectriques.

Fig. 7. Numbering convention of the four piezoelectric stack quadrupole.

des signaux sur l'oscilloscope servant de centrale d'acquisition de données est de $500 \mathrm{kHz}$. Selon les matrices d'essais proposées précédemment, il est intéressant dans un premier temps de valider la réciprocité de l'excitation, en comparant par exemple les couples E1R2 et E2R1 qui sont identiques, tout comme les couples E1R4 et E4R1 (Fig. 8). Le temps de vol est déterminé au sommet de la première alternance positive $\mathrm{du}$ « burst $»$ et de la première alternance du signal de réception. La différence de ces deux mesures correspond au temps de vol et la distance est mesurée entre chaque sommet de chaque pyramide afin de déterminer la vitesse de l'onde guidée. 


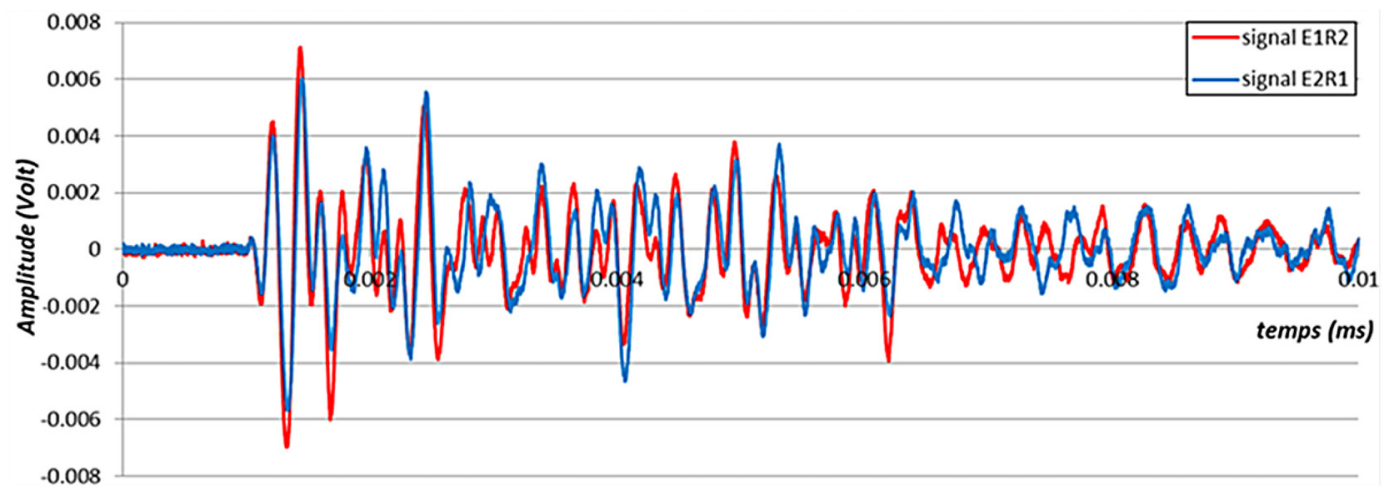

Fig. 8. Validation de la réciprocité de l'excitation ultrasonore sur une plaque en aluminium.

Fig. 8. Validation of the reciprocity of the ultrasonic waves propagating in an aluminum sheet.

Ainsi, pour la plaque en tôle d'aluminium, dans le sens E1R2 ou E2R1, qui correspond au sens perpendiculaire au sens de laminage, les vitesses mesurées sont de l'ordre de 589 et $593 \mathrm{~m} / \mathrm{s}$, alors que dans le sens du laminage (couples E3R2 ou E2R3), elles sont respectivement de 653 et $664 \mathrm{~m} / \mathrm{s}$. Par contre, dans le sens E1R3 ou E3R1 les vitesses estimées sont respectivement de 720 et $704 \mathrm{~m} / \mathrm{s}$. La sensibilité à l'anisotropie du matériau est ainsi mesurable puisque par rapport à la valeur de vitesse la plus basse (mesurée pour le couple E1R2), des augmentations de vitesse de 8,3\% et de 16,7\% sont mesurées pour les directions E2R3 et E2R4. Il est intéressant de comparer ce résultat avec le coefficient de Lankford, caractéristique de l'anisotropie d'une tôle d'aluminium obtenue par laminage. Ce coefficient, mesuré à partir d'essais de traction réalisés sur des éprouvettes dans le sens $0^{\circ}$ (sens de laminage) et dans le sens $90^{\circ}$ (direction perpendiculaire au sens de laminage), correspond au rapport entre la déformation transverse et la déformation en épaisseur de l'éprouvette. Pour une tôle d'aluminium, il varie de 0,82 dans le sens de laminage à 0.58 dans le sens perpendiculaire [13].

Dans la tôle en laiton, la moyenne des vitesses des ondes associées aux couples E1R2, E2R1, E3R4 ou E4R3 est de $446 \mathrm{~m} / \mathrm{s}$, avec un écart-type de l'ordre de $8 \mathrm{~m} / \mathrm{s}$. Dans le sens perpendiculaire, la vitesse moyenne correspondant aux couples E1R4, E4R1, E2R3 et E3R2 est très peu différente, de l'ordre de $495 \mathrm{~m} / \mathrm{s}$, avec un écart-type de $2 \mathrm{~m} / \mathrm{s}$. Enfin, dans le sens transverse E1R3, la vitesse est de $481 \mathrm{~m} / \mathrm{s}$, avec un écart-type de l'ordre de $6 \mathrm{~m} / \mathrm{s}$. Les écarts de vitesse par rapport à la vitesse la plus faible (couple E1R2) sont alors de 9,9\% dans le sens E2R3 et de $7,8 \%$ dans le sens E1R3, soit une sensibilité moindre à l'anisotropie que la plaque en tôle d'aluminium.

\section{Conclusion}

La nanoindentation instrumentée est généralement assimilée à un essai mécanique quasi-statique. La méthode présentée dans cet article est une mesure en dynamique qui repose sur la génération des ondes ultrasonores guidées avec un indenteur vibrant à une fréquence de plusieurs kilohertz, très supérieure à l'indentation en mode CSM. Cette démarche expérimentale nouvelle permet de réaliser une inspection et une caractérisation non-destructives de pièces de structure. Cette méthode couplée à la vibrométrie Laser a permis d'identifier la propagation des ondes dans les plaques et de localiser les défauts dans une structure. Il est alors apparu possible de disposer quatre indenteurs sur une structure pour pouvoir déterminer des vitesses de propagation dans différentes directions afin de quantifier l'anisotropie de la structure et, à terme, ses propriétés élastiques.

Cette nouvelle approche n'a pas pour objectif de remplacer la nanoindentation instrumentée mais au contraire d'en élargir le champ d'application. L'utilisation de ce type de caractérisation sur une structure réelle d'un aéronef est une perspective intéressante. Elle pourrait nécessiter le développement de systèmes équivalents avec des indenteurs de formes diverses, par exemple à géométrie sphérique [14].

Remerciements. Les auteurs remercient Daniel Osmont et Marc Dupont pour l'apport de leurs connaissances sur les techniques ultrasonores et les ondes de Lamb, ainsi que Michel Bejet et Patrick Caro pour leur contribution à la réalisation des démonstrateurs.

\section{Références}

[1] W.C.Oliver, G.M.Pharr, AIP Conference proceedings 7 (1992) 1564-1583

[2] M.F.Doerner, W.D.Nix, J. Mater. Res. 1 (1986) 601-609

[3] J.L. Loubet, J.M. Georges, G. Meille, Vickers indentation curves of elastoplastic materials, in American Society for Testing and Materials STP 889, Microindentation Techniques in Materials Science and Engineering, P.J.Blau, B.R.Lawn eds, 1986, pp. 72-89

[4] S.A.S. Asif, K.J. Wahl, R.J. Colton, Rev. Sci. Instrum. 70 (1999) 2408-2413

[5] B.Boro Djordjevic, Quantitative ultrasonic guided wave testing of composites, The 39th Annual Review of Progress, 2013 
[6] K. Diamanti, C. Soutis, J.M. Hodgkinson, Lamb waves for the non-destructive inspection of monolithic and sandwich composite beams, in Composites Part A : Applied Science and anufacturing, Vol. 36, 2005, pp. 189-19

[7] D. Osmont, D. Devillers, F. Taillade, Health monitoring of sandwich plates based on the analysis of the interaction of Lamb waves with damages, in Proceedings of SPIE Smart Structures and Materials : Smart Structures and Integrated Systems, 2001, pp. 290-301

[8] B. Lamboul, F. Passilly, J.M. Roche, D. Balageas, AIP Conference Proceedings 1650 (2014) 319

[9] J.-C. Krapez, F. Taillade, D.L. Balageas, Quantitative InfraRed Thermography Journal 2 (2005) 191-206

[10] S.D. Holland, C. Uhl, Z. Ouyang, et al., NDTEEE Int. 44 (2011) $775-782$
[11] G. Guillonneau, Nouvelles techniques de nanoindentation pour des conditions expérimentales difficiles : très faibles enfoncements, surfaces rugueuses, température, Thèse de doctorat de l'École Centrale de Lyon, 2012

[12] B. Lamboul, B. Passilly, J.M. Roche, D. Osmont, AIP Conference proceedings 1511 (2013) 1003

[13] Y. Chastel, V. Nalewajk, R. Forestier, E. Massoni, Caractérisation du comportement anisotrope de tôles d'aluminium, Groupe Français de Rhéologie, $40^{\mathrm{e}}$ colloque annuel du Groupe Français de Rhéologie, Oct 2005, Nice, France. hal-00578632

[14] F.L. Degertekin, B.T. Khuri-Yakub, J. Acoust. Soc. Am. 99 (1996) 299-308 